\title{
Dental and periodontal disease in hemodialysis patients is associated with inflammation; a study from North India
}

\author{
Lokesh Sharma $^{1}$, Raj Kumar Sharma ${ }^{2 * \mathbb{(}}$, Vamsi K Reddy ${ }^{1}$, Sonia Mehrotra ${ }^{2}$, Devina Pradhan ${ }^{3}$, Amit \\ Gupta $^{2}$, Narayan Prasad ${ }^{2}$, Dharmendra S. Bhadauria ${ }^{2}$, Anupama Kaul ${ }^{2}$, Sabyasachi Saha ${ }^{1}$ \\ ${ }^{1}$ Department of Public Health Dentistry, Sardar Patel Post Graduate Institute of Dental and Medical Sciences, Lucknow, India \\ ${ }^{2}$ Department of Nephrology, Sanjay Gandhi Post Graduate Institute of Medical Sciences, Lucknow, India \\ ${ }^{3}$ Department of Public Health Dentistry, Rama Dental College Hospital and Research Centre, Kanpur, Uttar Pradesh, India
}

\section{A R T I C L E I N F O}

\section{Article Type:}

Original

\section{Article History:}

Received: 5 February 2018

Accepted: 10 June 2018

Published online: 13 July 2018

\section{Keywords:}

Chronic kidney disease

Periodontitis

C-reactive protein

\begin{abstract}
A B S T R A C T
Introduction: Poor oral health and periodontitis is an important problem in chronic kidney disease (CKD) patients on hemodialysis (HD) awaiting kidney transplantation. This could contribute to increased morbidity and mortality as a result of infections and consequences of inflammation.

Objectives: To study the oral health status and assess its impact in inducing inflammation in CKD patients on HD.

Patients and Methods: Fifty CKD patients on HD (group I) were compared with 50 healthy controls (group-II) in a prospective cohort study. Oral health assessment was done with the WHO oral health assessment form with a mouth mirror and a community periodontal index (CPI) probe by a trained dentist. The demographic clinical data of oral health and periodontitis was collected in dialysis patients and healthy controls. CRP levels were assayed as markers of inflammation.

Results: Data shows that patients on dialysis have evidence of increased inflammation as indicated by raised CRP values. Our study shows the poor oral and mouth hygiene and periodontitis and inflammation was much more in dialysis patients as compared to control group $(P<0.05)$. Amongst dialysis patients with higher inflammation (CRP values), there was poorer oral health and more periodontitis $(P<0.05)$. This indicates that higher inflammation could result from poor dental hygiene and periodontitis.

Conclusion: Poor oral and mouth hygiene was associated with inflammation as indicated by high CRP values in CKD patients on dialysis with poor dental hygiene. Periodontitis was significantly associated with development of inflammation.
\end{abstract}

Implication for health policy/practice/research/medical education:

Oral health assessment is often an overlooked problem in chronic kidney disease patients on hemodialysis. This could have an adverse effect on outcome after dialysis and transplantation.

Please cite this paper as: Sharma L, Sharma RK, Reddy VK, Mehrotra S, Pradhan D, Gupta A, et al. Dental and periodontal disease in hemodialysis patients is associated with inflammation; a study from North India. J Renal Inj Prev. 2018;7(4):301-306. DOI: $10.15171 /$ jrip.2018.66.

\section{Introduction}

Inflammation is common among patients with chronic renal failure (CRF) $(1,2)$. Recent evidence suggests a high occurrence of periodontitis in occurrence patients (3), and patients on dialysis (4). The pro-inflammatory effect of periodontal disease is suggested by the association of periodontitis with markers of inflammation in patients with CKD. In chronic kidney disease (CKD) patients there is association of periodontitis, a potentially significant source of chronic inflammation, with elevation in C-reactive protein (CRP) levels (5).

Common symptoms of periodontal disease include tooth loss, gingivitis with gum inflammation and bleeding, excess tartar, infection, decay, tooth mobility, and gum recession with bone loss which can be identified by dentists. Periodontal disease is associated with elevations 
of several markers of chronic inflammation $(6,7)$. There is evidence that in CKD patients, chronic inflammation is associated with peridontitis. This is independent of other factors causing inflammation.

Studies have reported that moderate to severe periodontitis can precipitate an acute-phase response (8) and that effective intervention to treat periodontal therapy may be able to decrease systemic markers of inflammation (9). CRP elevation is a part of the acute phase response to acute and chronic inflammation. Many epidemiological studies have shown that serum CRP levels were elevated in patients with chronic periodontitis. CRP elevation is suggestive of inflammation and infection in the periodontitis, also occur in malnutrition and renal dysfunction (10). Proper periodontal therapy could be effective in reducing CRP level because it reduces bacterial load and inflammation. This can improve antibody response to various pathogens and result to decrease in local inflammation, with significant improvement in clinical parameters and reduction in the elevated CRP levels in patients with periodontitis (11-13). The poor oral health in CKD patients is an important problem, which may require a close collaboration between dentists and nephrologists (14).

\section{Objectives}

To study the oral health status and assess its impact in inducing inflammation in CKD patients on $\mathrm{HD}$.

\section{Patients and Methods}

\section{Study design}

This was the retrospective cohort study and the subjects were enrolled from the dialysis unit of a tertiary care center (SGPGIMS) in Lucknow, North India. Total of 50 participants were included in the group I, (CKD patients on dialysis). Fifty (age and sex matched) healthy controls (group II) were evaluated for oral and dental health. Various demographic and clinical variables were compared between CKD patients on dialysis (group I) with health controls (group II). CRP levels were assayed on a biochemistry analyzer (Beckman Coulter AU480) by latex particle immunoturbidimetric method.

All participants had an oral health assessment done with the WHO oral health assessment form (2013). Oral health assessment was performed by a trained dentist with a mouth mirror and a community periodontal index (CPI) probe to look for dental caries and missing teeth.

Periodontal status was assessed with the gingival bleeding score, pocket depth (PD) score and clinical loss of attachment (CAL) score. Gingival bleeding was scored as 0 (absent) or 1 (present). Probing depth was coded as 0 (absence of condition), 1 (pocket 4-5 mm) or 2 (pocket 6 $\mathrm{mm}$ or more). CAL was scored as 0 (0-3 mm), 1 (4-5 mm), 2 (6-8 mm), 3 (9-11 $\mathrm{mm})$ or 4 (12 $\mathrm{mm}$ or more). Periodontitis was defined by the case definition as detailed in the publication by Page and Eke (15) (Table 1).

\section{Ethical issues}

The research followed the tenets of the Declaration of Helsinki; informed consent was obtained from all patients. This study was approved by Ethics Committee of Sanjay Gandhi Post Graduate Institute of Medical Sciences, Lucknow, India (grant \# 2016-83-IP-EXP). All participants were informed about the objectives of the study and assured that the information will remain confidential. Participants also signed out consent forms.

\section{Statistical analysis}

For normally distributed, data were presented in mean \pm standard deviation (SD), minimum and maximum while non-normal data/discrete data were presented by median (inter-quartile range "IQR"), minimum and maximum. Means were compared among two groups (CKD and control groups) using one-way ANOVA while median values were compared by using Kruskal-Wallis $\mathrm{H}$ test. Various comparisons were performed to identify the actual significant pairs. Pearson's chi-squared test $(\chi 2)$ was used to compare the proportions between CKD and control groups. Wilcoxon signed rank test was used when data was in quantitative/ordinal scale. In case, scale was nominal, with two mutually exclusive possibilities, chisquare test was used to test the difference in proportions between the observations ( $P$ value less than 0.05 was considered as statistically significant). Statistics software, version-23 (SPSS-23, IBM, and Chicago, USA) used for data analysis.

\section{Results}

In the present study, total of 50 participants were included, in the group1 (CKD patients on dialysis), group II included 50 healthy controls. Various clinical and demographic variables were compared between $\mathrm{CKD}$ patients and normal controls. Age range of the patients was between 15-60 years with mean age of the patients in the group I and group II was $36.2 \pm 11.2$ and $35.9 \pm 6.6$ years. One-way ANOVA test revealed that mean difference between the

Table 1. Case definition for periodontitis

\begin{tabular}{lll}
\hline Case definition & Clinical attachment loss & Probing depth \\
\hline Severe periodontitis & $\geq 2$ interproximal sites with CAL $\geq 4 \mathrm{~mm}$ (not on the same tooth) & And $\geq 1$ interproximal sites with PD $\geq 5 \mathrm{~mm}$ \\
Moderate periodontitis & $\geq 2$ interproximal sites with CAL $\geq 4 \mathrm{~mm}$ (not on the same tooth) & $\begin{array}{l}\text { OR } \geq 2 \text { interproximal sites with PD } \geq 5 \mathrm{~mm} \text { (not on } \\
\text { the same tooth) }\end{array}$ \\
No or mild periodontitis & Neither "moderate" or "severe" & Neither "moderate" or "severe"
\end{tabular}

PD, pocket depth. 
groups was not statistically significant $(P=0.4)$ which is an indication there was no confounding effect of the age on the significant difference if observed in the clinical variables between the groups.

CKD patients with healthy controls: the data collected from the group I (50 CKD patients on dialysis) and group II (50 healthy controls) was compared to test the significance levels. In Table 2, the type and degree of teeth involvement along with other clinical variables in group I was compared with control group (group II). As data was highly non-normally distributed, Mann Whitney $\mathrm{U}$ test was used to compare between the CKD patients with healthy controls for each of the variables. In the CKD patients (group I), lowest and highest number of teeth involving in the patients was observed ( 0 and 5 respectively). In the CKD group I, 25\% patients had not a single tooth involved, $50 \%$ patients had less than 2 teeth involved, $75 \%$ patients had up to 3 teeth involved. In the control group, $50 \%$ had not a single tooth involved,and $75 \%$ had only 1 or no tooth involvement. The distribution of the no of teeth involvement between 2 groups was statistically significantly different $(P<0.05)$ (Table 2). There was significant difference between CKD patients and healthy controls for the variables namely; DMF (Decayed missing filled permanent teeth), bleeding,
Pocket $4-5$, Pocket $\geq 6$, LOA (loss of attachment) $=0-3$, $\mathrm{LOA}=4-5$ and oral mucosal lesions $(P<0.05)$ while it was statistically insignificant between two groups for the variables; $\mathrm{M}, \mathrm{F}$ and $\mathrm{LOA} \geq 6(P>0.05)$. Mann Whitney $\mathrm{U}$ test was used to compare the mean ranks of the data (not mean or median). Despite same median and interquartile value of the variable "Pocket $\geq 6$ " for the CKD and control groups, result came out to be significant which revealed that distributions between two groups are statistically significant $(P<0.05)$. None of the values were between 0 to third quartile value and all the values in the groups were above the third quartile value (Table 2).

In Table 3, distribution of the categorical variables was compared between CKD (group I) and control group (group II). Pearson's chi-squared test was used to test the association between the study groups for the categorical variables. Our data shows indicated that there was significant difference in the proportions of males and females between dialysis patients and healthy controls $(P<0.05)$. Similarly, proportions were significantly different between the groups for the variables; fluorosis and erosion but proportions of the location, calculus, plaque and stains were not significantly different between the two groups $(P>0.05)$.

Our data shows that patients on dialysis have evidence of

Table 2. Demographic and clinical variables in the CKD and the control groups

\begin{tabular}{|c|c|c|c|c|c|}
\hline \multirow{2}{*}{ Clinical values } & \multicolumn{2}{|c|}{ CKD group $(n=50)$} & \multicolumn{2}{|c|}{ Control group $(n=50)$} & \multirow{2}{*}{$P$ value } \\
\hline & Mean \pm SD/ Median (IQR) & Min-Max & Mean \pm SD/ Median (IQR) & Min-Max & \\
\hline D & $1.5(0.0-3.0)$ & $0-5$ & $0.0(0.0-1.0)$ & $0-3$ & 0.029 \\
\hline M & $0.0(0.0-1.0)$ & $0-3$ & $0.0(0.0-0.0)$ & $0-2$ & 0.052 \\
\hline $\mathrm{F}$ & $0.0(0.0-1.3)$ & $0-4$ & $0.0(0.0-0.3)$ & $0-3$ & 0.781 \\
\hline DMF & $3.0(0.0-4.0)$ & $0-7$ & $2.0(0.0-3.0)$ & $0-5$ & 0.008 \\
\hline Bleeding & $0.0(0.0-0.5)$ & $0-8$ & $0.0(0.0-0.0)$ & $0-5$ & 0.011 \\
\hline Pocket 4-5 & $0.0(0.0-4.25)$ & $0-8$ & $0.0(0.0-0.0)$ & $0-7$ & 0.035 \\
\hline Pocket $\geq 6$ & $0.0(0.0-0.0)$ & $0-5$ & $0.0(0.0-0.0)$ & $0-0$ & 0.042 \\
\hline LOA 0-3 & $6.0(5.0-6.0)$ & $4-6$ & $6.0(3.0-6.0)$ & $2-6$ & 0.009 \\
\hline LOA 4-5 & $0.0(0.0-1.0)$ & $0-2$ & $0.0(0.0-0.0)$ & $0-1$ & 0.001 \\
\hline $\mathrm{LOA} \geq 6$ & $0.0(0.0-0.0)$ & $0-1$ & $0.0(0.0-0.0)$ & $0-0$ & 0.080 \\
\hline Oral macro & $0.0(0.0-0.0)$ & $0-5$ & $0.0(0.0-0.0)$ & $0-0$ & 0.006 \\
\hline
\end{tabular}

Mann-Whitney $U$ test used to compare between Intervention and control groups.

Data presented in median (IQR), where IQR= Inter-quartile range showing first and third quartile Min-Max showing minimum and maximum No. of teeth involved in the group. $P<0.05$ significant

Table 3. Distribution of the clinical characteristics in the CKD and the control groups

\begin{tabular}{|c|c|c|c|c|}
\hline Variables & & $\begin{array}{c}\text { CKD group }(n=50) \\
\text { No. }(\%)\end{array}$ & $\begin{array}{c}\text { Control }(n=50) \\
\text { No. (\%) }\end{array}$ & $P$ value* \\
\hline Gender & Male & $47(94.0)$ & $5(10.0)$ & $<0.001$ \\
\hline Location & Urban+ Peri-urban & $44(88.0)$ & $39(78.0)$ & 0.183 \\
\hline Fluorosis & Present & $19(38.0)$ & $0(0.0)$ & $<0.001$ \\
\hline Erosion & Present & $20(40.0)$ & $0(0.0)$ & $<0.001$ \\
\hline Calculas & Present & $4(8.0)$ & $7(14.0)$ & 0.338 \\
\hline Plaque & Present & $8(16.0)$ & $6(12.0)$ & 0.564 \\
\hline Stain & Present & $21(42.0)$ & $16(32.0)$ & 0.300 \\
\hline
\end{tabular}

* Pearson chi-square test, $P<0.05$ significant. 
Table 4. Comparison between the two groups

\begin{tabular}{lccc}
\hline Variables & $\begin{array}{c}\text { Control } \\
\text { group }\end{array}$ & CKD group & $\begin{array}{c}\boldsymbol{P} \\
\text { values }\end{array}$ \\
\hline CRP levels (mg/L) & $1.03 \pm 0.78$ & $10.22 .83 \pm 4$ & 0.004 \\
Age (y) & $35.9 \pm 6.64$ & $36.2 \pm 11.1$ & 0.417 \\
DMF (score) & 126 & 96 & 0.008 \\
Periodontitis (score) & 46 & 188 & 0.000 \\
LOA (score) & 0 & 31 & 0.009 \\
Fluorosis (score) & 14 & 0 & 0.001 \\
Erosion (score) & 12 & 0 & 0.001 \\
Calculus (score) & 7 & 4 & 0.338 \\
Plaque (score) & 6 & 8 & 0.564 \\
Stains (score) & 16 & 21 & 0.300 \\
Oral-mucosal lesions (score) & 0 & 10 & 0.006 \\
\hline
\end{tabular}

increased inflammation as indicated by raised CRP values. Amongst dialysis patients with higher inflammation (CRP values), there was poorer oral health and significantly increased periodontitis score (Table 4). This indicates that higher inflammation could result from poor mouth, dental hygiene and periodontitis. Our study shows the poor oral and mouth hygiene and periodontitis causing inflammation were much more in dialysis patients compared to the control group.

\section{Discussion}

The present study was conducted to evaluate oral health and oral hygiene in CKD patient on hemodialysis (HD). A total of 50 participants on dialysis were included in our study. A study conducted by Brito et al (16) had patients with a mean age of 50 years. In this study there was no significant cofounding effect of age on the various clinical variables of oral health in the two groups.

In our present study, the number of teeth involved as a clinical variable was compared between CKD group and control group. The number of decayed teeth were higher in the CKD group which was statistically significant $(P=0.029)$. Our present study and the study done by Brito et al, revealed that bleeding on probing and the percentage sites with clinical attachment loss of $0-3 \mathrm{~mm}$ and 4-5 $\mathrm{mm}, \mathrm{PD}$ of $4-5 \mathrm{~mm}$ were found to be significantly higher ( $P=0.009, P=0.001, P=0.035$ respectively) in CKD group on dialysis (16).

CKD is progressive impairment of the kidney function that is associated with the deterioration of nephrons occurring as a consequence of diabetes, chronic glomerulonephritis and hypertension. Progression of irreversible CKD to endstage renal disease (ESRD) requires renal replacement therapy (dialysis or kidney transplantation) $(17,18)$.

Patients having $\mathrm{CKD}$ are prone to develop various pathologic alterations in the oral cavity. A variety of oral signs and symptoms like xerostomia, dental caries, periodontal disease, pockets, dental fluorosis, erosion and oral mucosal lesions have been reported in CKD patients on dialysis (19). Our present study shows that patients on hemodialysis had significantly higher levels of decayed teeth $(P=0.029)$, bleeding on probing $(P=0.011)$, pockets with depth of 4-5 mm $(P=0.035)$, clinical attachment loss of $0-3 \mathrm{~mm}, 4-5 \mathrm{~mm}(P=0.09$ and $P=0.01$ respectively) and oral mucosal lesions $(P=0.006)$.

A study conducted by Nylund et al (19) reported that the prevalence of periodontal disease ( $P D \geq 4 \mathrm{~mm}$ ) was found to be higher in CKD patients (88\%). Other studies conducted by Ahmed et al (20), Sharma et al (21), and Jyothi et al (22), indicated that periodontal destruction suggested by CAL score was higher amongst the patients of CKD.

In our present study, results show that there was significant difference in the dental fluorosis and erosion scores $(P=0.001)$ whereas no significance was found in calculus, plaque and stain scores $(P=0.338, P=0.564$ and $P=0.300$ respectively) amongst $\mathrm{CKD}$ patients group compared to healthy control group. However, higher scores were found in $\mathrm{CKD}$ group amongst the plaque and stains variable when compared with the control group. Accordingly, a study conducted by Bots et al (23) revealed that patients had significantly more teeth covered with calculus than the control group patients $(P<0.05)$.

Periodontal disease is believed to be associated with cardiovascular disease and systemic atherosclerosis. Intervention studies have shown that the treatment of periodontal disease improves serum inflammatory markers and flow mediated arterial dilation, a marker of endothelial function that becomes dysfunctional early in the case of atherosclerosis (24-28). The data collected from the group 1 (CKD patients) was compared with the control (group 2). The results of our study revealed that there was a statistically higher score in dialysis patients (group 1) in the following variables; bleeding on probing, PD score of 4-5 mm, LOA 0-3mm, and 4-5 mm ( $P=0.05)$. These results are consistent with other reports of oral lesions in CKD patients $(20,29)$.

Recent reports have indicated that patients with severe periodontitis have raised serum levels of CRP, when compared with normal healthy population $(30,31)$ There is a significant deterioration of oral and periodontal health in patients with CKD compared to healthy controls which is associated with higher CRP levels, which indicates inflammation. This may require remedial intervention $(32,33)$.

\section{Conclusion}

Our study confirms that poor oral and periodontal health is common in CKD patients on dialysis. There was significant association between periodontitis, poor oral health and raised CRP levels. This indicates increased inflammation and poor dental health in dialysis patients as compared to healthy control group. Periodontitis diagnosis and management need to be better addressed in dialysis patients. This would need increasing awareness amongst nephrology community as patients on dialysis may need 
regular dental check up to improve the oral and dental health with the help of dental community. The treatment of periodontitis could translate into improvement of inflammation and reduction in the harmful inflammatory response. The oral disease is quite prevalent in patients with advanced CKD. Good oral hygiene could have a favorable effect by reducing and preventing inflammation. Our study suggests that oral health check up should be included as a routine pre-transplant evaluation .

\section{Limitation of the study}

The main limitation of this study was the small sample size.

\section{Authors' contribution}

LS, RKS, VKR, SM, DP, AG, NP, DSB, AKandSS participated in research design. LS, RKS and SM participated in the writing of the paper. LS, RKS, VKR, SM, DP, AG, NP, DSB, $A K$ and SS participated in performance of the research. LS, RKS and SM contributed to new reagents or analytic tools. LS, RKS, and SM participated in data analysis.

\section{Conflicts of interest}

The authors declare no conflict of interest.

\section{Ethical considerations}

Ethical issues (including plagiarism, data fabrication, double publication) have been completely observed by the authors.

\section{Funding/Support}

This work was supported by grants from Intramural Research funds from Sanjay Gandhi Post Graduate Institute of Medical Sciences, Lucknow India (Grant \# 2016-83-IP-EXP).

\section{References}

1. Bergstrom J, Lindholm B. What are the causes and consequences of the chronic inflammatory state in chronic dialysis patients. Semin Dial, 2000;13:163-4. doi: 10.1046/j.1525-139x.2000.00044.x

2. Kaysen GA. The microinflammatory state in uremia: Causes and potential consequences. J Am Soc Nephrol. 2001;12:1549-57.

3. Kshirsagar AV, Moss KL, Elter JR, Beck JD, Offenbacher S, Falk RJ. Periodontal disease is associated with renal insufficiency in the Atherosclerosis Risk In Communities (ARIC) study. Am J Kidney Dis. 2005;45:650-7.

4. Rahmati MA, Craig RG, Homel P, Kaysen GA, Levin NW. Serum markers of periodontal disease status and inflammation in hemodialysis patients. Am J Kidney Dis. 2002;40:9839. doi: 10.1053/ajkd.2002.36330

5. Chen LP, Chiang CK, Chan CP, Hung KY, Huang CS. Does periodontitis reflect inflammation and malnutrition status in hemodialysis patients? Am J Kidney Dis. 2006;47:815-22. doi: 10.1053/j.ajkd.2006.01.018

6. Noack B, Genco RJ, Trevisan M, Grossi S, Zambon JJ, De Nardin E. Periodontal infections contribute to elevated systemic C-reactive protein level. J Periodontol.
2001;72:1221-7. doi: 10.1902/jop.2000.72.9.1221.

7. Slade GD, Offenbacher S, Beck JD, Heiss G, Pankow JS. Acute-phase inflammatory response to periodontal disease in the US population. J Dent Res. 2000;79(1):49-57. doi: $10.1177 / 00220345000790010701$.

8. Ebersole JL, Cappelli D. Acute-phase reactants in infections and inflammatory diseases. Periodontol. 2000;23:19-49.

9. D'Aiuto F, Parkar M, Andreou G, Suvan J, Brett PM, Ready D, Tonetti MS: Periodontitis and systemic inflammation: Control of the local infection is associated with a reduction in serum inflammatory markers. J Dent Res. 2004;83:156160. doi: $10.1177 / 154405910408300214$.

10. Gomes-Filho IS, Freitas Coelho JM, da Cruz SS, Passos JS, Teixeira de Freitas CO, Aragão Farias NS, et al. Chronic periodontitis and C-reactive protein levels. J Periodontol. 2011;82:969-78. doi: 10.1902/jop.2010.100511.

11. Noack B, Genco RJ, Trevisan M, Grossi S, Zambon JJ, Nardin ED. Periodontal infections contribute to elevated systemic C-reactive protein level. J Periodontol. 2001;72:1221-7. doi: 10.1902/jop.2000.72.9.1221.

12. Kumar S, Shah S, Budhiraja S, Desai K, Shah C, Mehta D. The effect of periodontal treatment on C-reactive protein: A clinical study. J Nat Sc Biol Med. 2013;4:379-82. doi: 10.4103/0976-9668.116991.

13. Ramamoorthy RD, Nallasamy V, Reddy R, Esther N, Maruthappan Y. A review of C-reactive protein: A diagnostic indicator in periodontal medicine. J Pharm Bioall Sci. 2012;4:422-26. doi: 10.4103/0975-7406.100318.

14. Borawski J, Wilczynska-Borawska M, Stokowska W, Mysliwiec M. The periodontal status of pre-dialysis chronic kidney disease and maintenance dialysis patients. Nephrol Dial Transplant. 2007;22:457-464. doi: 10.1093/ndt/gfl676.

15. Page RC, Eke PI. Case Definitions for Use in PopulationBased Surveillance of Periodontitis. J Periodontol. 2007;78:1387-99. doi: 10.1902/jop.2007.060264.

16. Brito F, Almeida S, Figueredo C, Bregman R, Suassuna J, Fischer R. Extent and severity of chronic periodontitis in chronic kidney disease patients. J Periodontol Res. 2012;47:426-30. doi: 10.1111/j.1600-0765.2011.01449.x.

17. Chen LP, Chiang CK, Chan CP, Hung KY, Huang CS: Does periodontitis reflect inflammation and malnutrition status in hemodialysis patients? Am J Kidney Dis.2006;47:81522. doi: 10.1053/j.ajkd.2006.01.018.

18. Proctor R, Kumar N, Stein A, Moles D, Porter S. Oral and dental aspects of chronic renal failure. J Dent Res. 2005;84:199-208. doi: 10.1177/154405910508400301

19. Nylund, K, Meurman J, Heikkinen A, Honkanen E, Vesterinen $\mathrm{M}$, Ruokonen $\mathrm{H}$. Oral health in predialysis patients with emphasis on periodontal disease. Quintessence Int. 2015;46:899-907. doi: 10.3290/j.qi.a34698

20. Tawig A, Jamal B, Eskandrani R, Al Dayel L, Al Twaijry S, Al Ghamdi E, et al. Assessment of periodontal disease severity among patients at different stages of chronic kidney disease. J Int Oral Health. 2016; 8(3):307-12. doi: 10.2047/jioh-0803-02

21. Sharma P, Dietrich T, Ferro C, Cockwell P, Chapple I. Association between periodontitis and mortality in stages 3-5 chronic kidney disease: NHANES III and linked mortality study. J Clin Periodontol. 2016;43:104-13. doi: $10.1111 /$ jcpe. 12502 .

22. Tadakamadla J, Kumar S, Mamatha G. Comparative evaluation of oral health status of chronic kidney disease (CKD) patients in various stages and healthy controls. Spec 
Care Dentist. 2014;34:122-126. doi: 10.1111/scd.12040.

23. Bots C, Poorterman J, Brand H, Kalsbeek H, Amerongen B, Veerman E, et al. The oral health status of dentate patients with chronic renal failure undergoing dialysis therapy. Oral Dis. 2006;12:176-80. doi: 10.1111/j.1601-0825.2005.01183.x

24. Menon V, Wang X, Greene T, Beck GJ, Kusek JW, Marcovina $\mathrm{SM}$, et al. Relationship between C-reactive protein, albumin, and cardiovascular disease in patients with chronic kidney disease. Am J Kidney Dis. 2003;42:44-52.

25. Chen L-P, Chiang C-K, Chan C-P, Hung K-Y, Huang C-S. Does periodontitis reflect inflammation and malnutrition status in hemodialysis patients? Am J Kidney Dis. 2006;47:815-22. doi: 10.1053/j.ajkd.2006.01.018

26. Rahmati MA, Craig RG, Homel P, Kaysen GA, Levin NW. Serum markers of periodontal disease status and inflammation in hemodialysis patients. Am J Kidney Dis. 2002;40(5):983-9. doi: 10.1053/ajkd.2002.36330

27. Mercanoglu F, Oflaz H, Oz O, Gökbuget AY, Genchellac $\mathrm{H}$, Sezer M, et al. Endothelial dysfunction in patients with chronic periodontitis and its improvement after initial periodontal therapy. J Periodontol. 2004;75:1694-00. doi: 10.1902/jop.2004.75.12.1694.

28. Favi E, Salerno MP, Romagnoli J, Castagneto M, Citterio F. Significant improvement in patient survival after renal transplantation in the last decade. Transplant Proc. 2011;43:285-7. doi: 10.1016/j.transproceed.2010.09.105

29. Kshirsagar AV, Craig RG, Moss KL, Beck JD, Offenbacher S, Kotanko P, et al. Periodontal disease adversely affects the survival of patients with end-stage renal disease. Kidney Int. 2009;75:746-51. doi: 10.1038/ki.2008.660.

30. Palmer S, Ruospo M, Wong G, Craig J, Petruzzi M, De Benedittis $\mathrm{M}$, et al. Patterns of oral disease in adults with chronic kidney disease treated with hemodialysis. Nephrol Dial Transplant. 2016;31:1647-53. doi: 10.1093/ndt/gfv413.

31. Gomes-Filho IS, Coelho JMF, Cruz SS, Passos JS, Freitas COT, Farias NSA, et al. Chronic periodontitis and C-reactive protein levels. J Periodontol. 2011;82:969-78. doi: 10.1902/jop.2010.100511

32. Dencheva M. Dialysis, Renal Transplantation and Oral Health-Many-Sided Nature of Dental Focal Doctrine. Biotechnology \& Biotechnological Equipment. 2010;24:1878-81. doi: 10.2478/V10133-010-0038-Y

33. Ruospo M, Palmer SC, Wong G, Craig JC, Petruzzi M, De Benedittis $\mathrm{M}$, et al. Periodontitis and early mortality among adults treated with hemodialysis: a multinational propensity-matched cohort study. BMC Nephrol. 2017;18(1):166. doi: 10.1186/s12882-017-0574-x.

Copyright (c) 2018 The Author(s); Published by Nickan Research Institute. This is an open-access article distributed under the terms of the Creative Commons Attribution License (http://creativecommons.org/licenses/by/4.0), which permits unrestricted use, distribution, and reproduction in any medium, provided the original work is properly cited. 